\title{
Remotal sets in tensor product spaces and $\varepsilon$-remotality
}

\author{
H. Salameh, R. Khalil* \\ Department of Mathematics, University of Jordan, Amman, Jordan.
}

\begin{abstract}
Let $X$ be a Banach space and $E$ a bounded set in $X$. For $x \in X$, we set $D(x, E)=\sup \{\|x-e\|: e \in E\}$. The set $E$ is called remotal if for any $x \in X$ there exists $e \in E$ such that $D(x, E)=\|x-e\|$. In this paper we prove some results on remotality in tensor product spaces. Further, we prove a main result "Every bounded set is $\varepsilon$-remotal", where the concept of $\epsilon$-remotality was introduced introduced in last couple of years and studied by many authors.
\end{abstract}

Keywords: Remotal sets, tensor product, Banach spaces, $\epsilon$-remotality.

2010 MSC: 41A28, 41A65.

(C)2019 All rights reserved.

\section{Introduction}

Let $X$ be Banach space and $E$ be a closed bounded subset of $X$. For $x \in X$ we set $D(x, E)=\sup \{\|x-e\|$ : $e \in E\}$. The set $E$ is called remotal in $X$ if for any $x \in X$, there exists $e_{0} \in E$ such that $D(x, E)=\left\|x-e_{0}\right\|$. The point $e_{0}$ is called the farthest point from $x$ in $E$. If the set of the farthest points in $E$ from $x$ is a singleton for all $x \in X$, then $E$ is called uniquely remotal.

The study of remotal sets started almost in the sixties. However, the study of remotal sets is not as rich as proximinal sets. One of the main conjectures in the theory of remotal sets is "A set $E$ in a Banach space $X$ is uniquely remotal if and only if $E$ is a singleton".

For Banach spaces $X$ and $Y$, we let $X{ }_{\otimes}^{\vee} Y$ and $X \hat{\otimes}_{\pi} Y$ denote the completed injective and the completed projective tensor products of $X$ and $Y$.

Remotality in tensor product spaces never been studied, unlike proximinality in tensor product spaces, $[4,5]$. In this paper we prove some results on remotality in both the projective and the injective tensor products.

The concept of $\epsilon$-remotality was introduced in [6], while the concept of $\epsilon$-proximinality was introduced in [9]. Many papers were written on such concepts, see [1-3]. For both concepts all papers assumed some conditions on a set to be $\epsilon$-remotal (or $\epsilon$ proximinal). In this paper we prove the main result "Every bounded set is $\epsilon$-remotal". We refer to $[7,8]$ for more on proximinality and remotality in different Banach spaces.

\footnotetext{
*Corresponding author

Email address: roshdi@ju.edu.jo (R. Khalil)
}

doi: $10.22436 /$ jmcs.019.02.05

Received: 2019-03-09 Revised: 2019-03-28 Accepted: 2019-04-03 


\section{Epsilon remotality in Banach spaces}

The notion of $\epsilon$-remotality was introduced in [1], and many results were obtained in that paper.

Definition 2.1. Let $X$ be a Banach space and $E \subseteq X$. Let $\epsilon>0$. We say $E$ is $\epsilon$-remotal if for every $x \in X$ there exist $x_{0} \in E$ such that $\left\|x-x_{0}\right\| \geqslant D(x, E)-\epsilon$.

In [1], many conditions were put so to prove that a set in a Banach space is $\epsilon$-remotal. In this section, our main result is: every bounded set is $\epsilon$-remotal.

Remark 2.2. We should remark that every remotal set is $\epsilon$-remotal. Indeed, let $E$ be remotal in $X$. If $x \in X$ and $x_{0}$ is the farthest point of $x$ in $E$, then for all $e \in E$

$$
\left\|x-x_{0}\right\| \geqslant\|x-e\| \geqslant\|x-e\|-\epsilon .
$$

However, the converse need not be true. Indeed, the set $(0,2]$ is not remotal in $\mathbb{R}$, but it is $\varepsilon$-remotal. Now, to show that the set is $\epsilon$-remotal, let $x \in \mathbb{R}$. Then

1. if $x \leqslant 1$, then 2 is the farthest point from $x$ in $(0,2]$;

2. if $x>1$, then for any $\epsilon>0$ take $x_{0} \in(0, \varepsilon)$, then

$$
\left\|x-x_{0}\right\| \geqslant\|x-e\|-\epsilon, \quad \forall e \in(0,2] .
$$

Hence, $(0,2]$ is $\varepsilon$-remotal in $\mathbb{R}$.

Definition 2.3. For $x \in X, \epsilon>0$, and $E \subseteq X$, we define $F_{\varepsilon}(x, E)=\{e \in E:\|x-e\|>D(x, E)-\epsilon\}$.

Note that $F(x, E) \subseteq F_{\varepsilon}(x, E)$ for every $\epsilon>0$.

Theorem 2.4. $\mathrm{F}_{\mathcal{\varepsilon}}(\mathrm{X}, \mathrm{E})$ is a closed set in $\mathrm{E}$ for every $\epsilon>0$.

Proof. Let $\left(e_{n}\right)$ be a sequence in $F_{\mathcal{\varepsilon}}(x, E)$ such that $e_{n} \rightarrow e$. We claim that $e \in F_{\varepsilon}(x, E)$.

Since $e_{n} \rightarrow e$, then $\lim _{n \rightarrow \infty}\left\|x-e_{n}\right\|=\|x-e\|$. But $\left\|x-e_{n}\right\| \geqslant D(x, E)-\epsilon$. Thus $\|x-e\|=\lim _{n \rightarrow \infty}\left\|x-e_{n}\right\|$ $\geqslant \mathrm{D}(x, \mathrm{E})-\epsilon$.

Hence $e \in F_{\varepsilon}(x, E)$.

Now we prove the main result of this section.

Theorem 2.5. Let $\mathrm{E}$ be any bounded set in a Banach space $\mathrm{X}$. Then for any $\epsilon>0, \mathrm{E}$ is $\varepsilon$-remotal in $\mathrm{X}$.

Proof. Let $\epsilon$ be arbitrary and $x \in X$. Let $r=D(x, E)=\sup \{\|x-e\|: e \in E\}$. If $E$ is remotal, then by Remark 2.2 , there is nothing to prove. So assume that $E$ is not remotal in $X$. Now, the set $K=B_{1}[x, r]-B_{1}[x, r-\epsilon]$ has a non-empty intersection with $E$. One can easily check that $K \cap E \neq \emptyset$. Since if not, then $r=D(x, E) \leqslant$ $r-\epsilon<r$. This is a contradiction. So, if we take any $x_{0} \in K \cap E$, then

$$
\left\|x-x_{0}\right\| \geqslant r-\epsilon=D(x, E)-\epsilon .
$$

Hence, $E$ is $\epsilon$-remotal in $X$.

\section{Remotality in injective and projective tensor product space}

Very few results are known in the injective tensor product of Banach spaces. In this section we prove some results on remotality in such spaces.

Definition 3.1 ([3]). A Banach space $X$ is said to have the approximation property if for every compact subset $K$ of $X$ and every $\epsilon>0$ there exists a finite rank operator $T: X \rightarrow X$ such that $\|T x-x\| \leqslant \epsilon$ for every $x \in K$. 
Lemma 3.2 ([3]). Let $\mathrm{X}$ and $\mathrm{Y}$ be Banach spaces such that $\mathrm{X}^{*}$ is reflexive and either $\mathrm{X}^{*}$ or $\mathrm{Y}^{*}$ has the approximation property. Then

$$
\left(X \stackrel{\vee}{\otimes}_{\epsilon} Y\right)^{*} \cong X^{*} \hat{\otimes}_{\pi} Y^{*}
$$

Lemma 3.3 ([3]). Let $\mathrm{X}$ and $\mathrm{Y}$ be reflexive Banach spaces such that $\mathrm{X}^{*}$ has the approximation property. If every operator from $\mathrm{X}$ to $\mathrm{Y}^{*}$ is compact, then

$$
\left(X \hat{\otimes}_{\pi} Y\right)^{*} \cong X^{*} \stackrel{\vee}{\otimes}_{\epsilon} Y^{*}
$$

Now we are ready to prove the following result which describes some remotal sets in the injective tensor product space.

Theorem 3.4. Let $\mathrm{X}$ be a reflexive space with the approximation property. If $\mathrm{H}$ is a finite dimensional subspace of $\mathrm{X}$, then $\mathrm{B}_{1}\left[\mathrm{X} \stackrel{\vee}{\otimes}_{\epsilon} \mathrm{H}\right]$ is remotal in $\mathrm{X} \stackrel{\vee}{\otimes}_{\epsilon} \mathrm{X}$.

Proof. Since $X$ is a reflexive space, then $X^{*}$ is also reflexive. Since $\mathrm{H}^{*}$ is finite dimensional, then $\mathrm{H}^{*}$ has the approximation property. Thus by Lemma 3.2 we have

$$
\left(X \stackrel{\vee}{\otimes}_{\epsilon} H\right)^{*} \cong X^{*} \hat{\otimes}_{\pi} H^{*}
$$

Now, since $X$ reflexive, then $X^{* *}=X$ has the approximation property. Further, any $T \in L\left(X^{*}, H^{* *}\right)=$ $\mathrm{L}\left(\mathrm{X}^{*}, \mathrm{H}\right)$ is compact, since $\mathrm{H}$ is finite dimensional. So, by Lemma 3.2 we get

$$
\left(X^{*} \hat{\otimes}_{\pi} H^{*}\right)^{*} \cong X \stackrel{\vee}{\otimes}_{\epsilon} H .
$$

Consequently, $X \stackrel{\vee}{\otimes}_{\epsilon} H$ is reflexive space. So, by Alaoglu Theorem $B_{1}\left[\left(X^{*} \hat{\otimes}_{\pi} H^{*}\right)^{*}\right]=B_{1}\left[X \stackrel{\vee}{\otimes}_{\epsilon} H\right]$ is compact in $w^{*}$-topology. Hence, $B_{1}\left[X \stackrel{\vee}{\otimes}{ }_{\epsilon} H\right]$ is remotal in $X \otimes_{\epsilon}^{\vee} X$.

\section{Remotality in projective tensor product space}

In this section we prove the following.

Theorem 4.1. Let $\mathrm{X}$ and $\mathrm{Y}$ be two Banach spaces and let $\mathrm{E}$ and $\mathrm{F}$ be two subsets of $\mathrm{X}$ and $\mathrm{Y}$, respectively. We let $[\mathrm{E}]$ and $[\mathrm{F}]$ denote the span of the sets $\mathrm{E}$ and $\mathrm{F}$, respectively. Assume $[\mathrm{E}]$ is a separable dual space in $\mathrm{X}$ and $[\mathrm{F}]$ is finite dimensional in $\mathrm{Y}$. Then every atom in $\mathrm{B}_{1}\left[[\mathrm{E}] \hat{\otimes}_{\pi}[\mathrm{F}]\right]$ has a farthest point in $\mathrm{X} \hat{\otimes}_{\pi} \mathrm{Y}$.

Proof. Let $f \in X, g \in Y$. Then

$$
\mathrm{D}\left(\mathrm{f} \otimes \mathrm{g}, \mathrm{B}_{1}\left[[\mathrm{E}] \hat{\otimes}_{\pi}[\mathrm{F}]\right]\right)=\sup _{w}\|\mathrm{f} \otimes \mathrm{g}-w\|, \quad w \in \mathrm{B}_{1}\left[[\mathrm{E}] \hat{\otimes}_{\pi}[\mathrm{F}]\right] .
$$

By the definition of supremum, there exists a sequence $\left(w_{m}\right) \in B_{1}\left[[E] \hat{\otimes}_{\pi}[F]\right]$ such that

$$
\mathrm{D}\left(\mathrm{f} \otimes \mathrm{g}, \mathrm{B}_{1}\left[[\mathrm{E}] \hat{\otimes}_{\pi}[\mathrm{F}]\right]\right)=\lim _{\mathrm{m} \rightarrow \infty}\left\|\mathbf{f} \otimes \mathrm{g}-w_{\mathrm{m}}\right\|, \quad w_{\mathrm{m}} \in \mathrm{B}_{1}\left[[\mathrm{E}] \hat{\otimes}_{\pi}[\mathrm{F}]\right] .
$$

Since $F$ is finite dimensional, then any element $k \in B_{1}\left[[E] \hat{\otimes}_{\pi}[F]\right]$ can be written as $k=\sum_{i=1}^{n} x_{i} \hat{\otimes}_{\pi} e_{i}$, where $x_{i} \in[E]$ and $\left\{e_{1}, e_{2}, \ldots, e_{n}\right\}$ is a basis for $F$. Thus, $w_{m}=\sum_{i=1}^{n} x_{i}^{m} \hat{\otimes}_{\pi} e_{i}$, where

$$
\begin{aligned}
& w_{1}=x_{1}^{1} \hat{\otimes}_{\pi} e_{1}+x_{2}^{1} \hat{\otimes}_{\pi} e_{2}+\cdots+x_{n}^{1} \hat{\otimes}_{\pi} e_{n}, \\
& w_{2}=x_{1}^{2} \hat{\otimes}_{\pi} e_{1}+x_{2}^{2} \hat{\otimes}_{\pi} e_{2}+\cdots+x_{n}^{2} \hat{\otimes}_{\pi} e_{n},
\end{aligned}
$$




$$
w_{k}=x_{1}^{k} \hat{\otimes}_{\pi} e_{1}+x_{2}^{k} \hat{\otimes}_{\pi} e_{2}+\cdots+x_{n}^{k} \hat{\otimes}_{\pi} e_{n} .
$$

Since, $w_{m} \in B_{1}\left[[E] \hat{\otimes}_{\pi}[F]\right]$ we have $\left\|w_{m}\right\| \leqslant 1$ is a bounded sequence. Now, consider the sequences $\left(x_{1}^{\mathrm{m}}\right),\left(x_{2}^{\mathrm{m}}\right), \ldots,\left(x_{\mathrm{n}}^{\mathrm{m}}\right)$.

Then by Helly's selection theorem each sequence of these sequences has a $w^{*}$-convergent subsequence because $[E]$ is a separable dual space in $X$.

Thus using a standard procedure, [2], we can extract $w^{*}$-convergent subsequences with uniform index, say $\left(x_{1}^{m_{j}}\right),\left(x_{2}^{m_{j}}\right), \ldots,\left(x_{n}^{m_{j}}\right)$.

Now, take the sequence

$$
u_{m_{j}}=x_{1}^{m_{j}} \hat{\otimes}_{\pi} e_{1}+x_{2}^{m_{j}} \hat{\otimes}_{\pi} e_{2}+\cdots+x_{n}^{m_{j}} \hat{\theta}_{\pi} e_{n} .
$$

Then $\left(u_{m_{j}}\right)$ is a subsequence of the sequence $\left(w_{n}\right)$ which is $w^{*}$-convergent, say to $w$. Then

$$
\lim _{m \rightarrow \infty}\left\|\mathbf{f} \otimes \mathbf{g}-w_{m}\right\|=D\left(f \otimes g, B_{1}\left[[E] \hat{\otimes}_{\pi}[F]\right]\right)=\|\mathbf{f} \otimes \mathbf{g}-w\| .
$$

Thus, every atom in $B_{1}\left[[E] \hat{\otimes}_{\pi}[F]\right]$ has a farthest point in $X \hat{\otimes}_{\pi} Y$.

Corollary 4.2. Let $\mathrm{Y}$ be a finite dimensional subspace of a Banach space $\mathrm{X}$. Then each atom in $\mathrm{B}_{1}\left[\ell^{\mathrm{p}} \hat{\otimes}_{\pi} \mathrm{Y}\right]$ has a farthest point in $\ell^{p} \hat{\otimes}_{\pi} X$ for $1<p<\infty$.

\section{Acknowledgment}

The authors would like to thank the referee for his thorough proof reading of the manuscripts and the corrections he suggested.

\section{References}

[1] M. Açikgoz̈, 2 - - -Proximinality in generalized 2-normed spaces, Int. Math. Forum, 5 (2010), 781-786. 1, 2, 2

[2] H. Alizadeh, S. Rezapour, S. M. Vaezpour, $\epsilon$-simultaneous approximations of downward sets, Bull. Iranian Math. Soc., 39 (2013), 193-203. 4

[3] A. Y. As'ad, A. M. Abu Ghazal, On e-proximinal and є-Chebyshev sets in normed spaces, Int. Math. Forum, 6 (2011), 1065-1070. 1, 3.1, 3.2, 3.3

[4] W. Deeb, R. Khalil, Best approximation in $\operatorname{Lp}(\mathrm{I}, \mathrm{X}), 0<\mathrm{p}<1$, J. Approx. Theory, 58 (1989), 68-77. 1

[5] D. Hussein, R. Khalil, Best approximation in tensor product spaces, Soochow J. Math., 18 (1992), 397-407. 1

[6] V. Indumathi, N. Prakash, E-proximinal subspaces, J. Math. Anal. Appl., 450 (2017), 1-11. 1

[7] R. Khalil, Best approximation in tensor products, Numer. Funct. Anal. Optim., 8 (1985/86), 347-356. 1

[8] R. Khalil, S. Al-Sharif, Remotal sets in verctor valued function spaces, Scientiae Mathematicae Japonicae Online, 63 (2006), 395-403. 1

[9] H. Mazaheri, M. Zarenejhad, Some new results on remotal points in normed spaces, J. Mahani Math. Res. Cent., 3 (2014), 37-50. 1 